\title{
Controle Psicológico Materno e Problemas Internalizantes em Pré-Escolares ${ }^{1}$
}

\author{
Taiane Lins ${ }^{2}$ \\ Patrícia Alvarenga \\ Universidade Federal da Bahia
}

\begin{abstract}
RESUMO - O estudo investigou as relações entre o controle psicológico e o controle comportamental materno e os problemas internalizantes de crianças entre 3 e 5 anos. Participaram 103 mães que responderam ao CBCL e à Entrevista Estruturada sobre Práticas Educativas Parentais e Socialização Infantil. Os resultados revelaram que duas das síndromes que representam o agrupamento de problemas internalizantes, ansiedade/depressão e retraimento, estiveram positivamente correlacionadas ao controle crítico, uma das dimensões do controle psicológico. É possível que o controle crítico materno limite o desenvolvimento socioemocional da criança e o acesso a reforçadores, contribuindo para o surgimento do retraimento e de sintomas de ansiedade/ depressão. Discute-se também a relevância de outras variáveis para a compreensão dos problemas internalizantes, como as práticas de socialização emocional.
\end{abstract}

Palavras-chave: problemas internalizantes, controle materno, desenvolvimento infantil

\section{Maternal Psychological Control and Internalizing Problems of Preschool Children}

\begin{abstract}
The present study investigated relations between maternal psychological control and behavior control and internalizing problems of preschool children, with ages varying between three and five years. Participants in the study were 103 mothers who completed the CBCL and the Child Socialization and Parental Childrearing Practices Structured Interview. The results showed that two of the syndromes representing the group of internalizing problems, anxiety/depression and withdrawal, were positively correlated with critical control, which is a dimension of psychological control. It is possible that maternal critical control limits the socio-emotional development of children and their access to reinforcers, contributing to the emergence of withdrawal and symptoms of anxiety/depression. The relevance of other variables for the understanding of internalizing problems, as emotional socialization practices, is also discussed.
\end{abstract}

Keywords: internalizing problems, maternal control, child development

O estudo dos problemas de comportamento internalizantes se intensificou a partir da década de 1960, quando esses problemas passaram a fazer parte de sistemas de avaliação com base empírica (Achenbach \& Edelbrock, 1978; Achenbach, 1966; Peterson, 1961). Contudo, na literatura, ainda não há uma definição precisa para esses problemas (Alvarenga, 2000; Anselmi, Piccinini, Barros, \& Lopes, 2004), o que se deve, em parte, a sua complexidade, e também ao amplo conjunto de fenômenos comportamentais que o conceito abrange. As definições encontradas na literatura geralmente se referem a uma operacionalização do construto baseada nos instrumentos do Sistema Achenbach de Avaliação Baseada Empiricamente (ASEBA) que avaliam problemas emocionais e comportamentais em diferentes fases do desenvolvimento, através do relato de diferentes informantes (CBCL - Achenbach \& Rescorla, 2000). De acordo com esse instrumento, os problemas internalizantes envolvem as síndromes reatividade emocional, ansiedade e depressão, queixas somáticas e retraimento.

1 Este estudo foi derivado da Dissertação da primeira autora, realizada sob a supervisão da segunda autora, a Profa Dra Patrícia Alvarenga. A Dissertação foi apresentada ao Programa de Pós-Graduação em Psicologia da Universidade Federal da Bahia, Salvador-BA

2 Endereço para correspondência: Programa de Pós-Graduação em Psicologia, UFBA, Rua Aristides Novis, 197, Federação, Salvador, BA, Brasil. CEP: 40210-909. E-mail: lins.taiane@gmail.com
De acordo com alguns estudos (ex. Anselmi et al., 2004; Sawyer et al., 2001), os problemas internalizantes apresentam prevalência, entre $12,8 \%$ e $15,2 \%$ e quando não são tratados podem evoluir para transtornos mentais como os Transtornos Ansiosos e os Transtornos de Humor (Kovacs \& Devlin, 1998). Entretanto, para Mathiesen, Sanson, Stoolmiller e Karevold (2009), não há consenso sobre os fatores específicos que contribuem para o desenvolvimento desses problemas e diferentes preditores têm sido identificados. A falta de consenso pode ser um reflexo, por exemplo, do fato de os problemas internalizantes envolverem quatro síndromes diferentes, as quais podem ter fatores etiológicos e trajetórias de desenvolvimento distintas. Contudo, de forma geral, características da própria criança, assim como variáveis ambientais parecem estar associadas aos problemas internalizantes.

Dentre os fatores individuais, estão o sexo da criança e as características temperamentais, como as dificuldades de adaptação, a inibição comportamental e o temor excessivo (Keiley, Bates, Dodge, \& Pettit, 2000; Keiley, Lofthouse, Bates, Dodge, \& Pettit, 2003). Em relação ao sexo da criança, os achados ainda não são conclusivos. Alguns estudos verificaram que as meninas apresentam mais problemas internalizantes, em comparação aos meninos (Crawford, Cohen, Midlarsky, \& Brook, 2001; Marturano, Toller, \& Elias, 2005; Peterson, 1961). Resultado divergente foi encontrado por Sawyer et al. (2001) ao avaliarem a prevalência dos 
problemas internalizantes entre crianças e adolescentes, entre 4 e 17 anos. Para os meninos, a prevalência foi de $14,5 \%$, enquanto entre as meninas foi de $11,1 \%$. Outras investigações, por outro lado, não identificaram diferenças na prevalência entre os sexos (Bayer, Hastings, Sanson, Ukoumunne, \& Rubin, 2010; Bayer, Sanson, \& Hemphill, 2009).

Como fatores ambientais, Oland e Shaw (2005) destacam os eventos de vida estressores, a pobreza, a rejeição de pares e a negligência, além dos fatores familiares, os quais possuem importância destacada. Entre as variáveis familiares, a ocorrência de conflito interparental, a presença de depressão materna ou outra psicopatologia parental, assim como a hostilidade e o alto controle parental, têm sido os fatores mais associados aos problemas internalizantes (Bayer, Sanson, \& Hemphill, 2006; McLeod, Weisz, \& Wood, 2007; McLeod, Wood, \& Weisz, 2007). Outro fator que tem se destacado são as práticas maternas para regular as emoções dos filhos (Silk et al., 2011). Através de análises de variância, Cova, Maganto e Melipillán (2005) verificaram maior frequência de problemas internalizantes em pré-adolescentes que sofriam mais adversidades no ambiente familiar, como a ausência de carinho entre pais e filhos, a discórdia entre adultos, a violência familiar e o controle inadequado. Essas relações foram particularmente significativas entre as meninas.

Apesar de diversos estudos investigarem diferentes práticas adotadas pelos pais para socializar os filhos, ainda não há evidências concretas de como essas variáveis se relacionam ao surgimento e à manutenção dos problemas internalizantes. Patterson, Reid e Dishion (1992) especulam que um caminho possível seria o uso, por parte dos pais, de desprezo, de indução de culpa e de ansiedade durante as interações com os filhos. Essas interações seriam mais frequentes "em famílias extremamente aversivas" (p. 77) que não permitem que os filhos desenvolvam confiança e se tornem independentes, adotando, por exemplo, expectativas muito elevadas e difíceis de serem alcançadas, que contribuem para a desvalorização do que os filhos fazem. Nesse sentido, Barber (1996) propõe um modelo segundo o qual os pais influenciam o desenvolvimento dos filhos através da adoção de duas formas distintas de controle: o controle psicológico e o controle comportamental. O controle psicológico envolve tentativas de interferir no funcionamento psicológico dos filhos, através da humilhação, da restrição da expressão verbal infantil, da indução de culpa, da retirada de amor e da invalidação de sentimentos (Barber, 1996). McShane e Hastings (2009) avaliam essa forma de controle através de duas dimensões: o controle crítico (ex. críticas negativas constantes, ironia, rejeição) e a superproteção parental (ex. demonstrações de afeto excessivas ou em momentos inadequados, restrição da independência da criança para a exploração do ambiente). Por outro lado, o controle comportamental refere-se ao emprego de estratégias de controle do comportamento da criança, as quais envolvem a sinalização das consequências dos comportamentos infantis, a sugestão de alternativas, as explicações sobre os motivos para a imposição de determinada regra, além de monitoramento e definição de limites. Essa forma de controle é avaliada por McShane e Hastings através do suporte apropriado que envolve, por exemplo, a orientação e a estimulação parental para os filhos interagirem com pares.

Caron, Weiss, Harris e Catron (2006) sugerem que o controle psicológico se relaciona aos problemas internalizantes, principalmente, porque essa forma de controle tende a restringir a autonomia da criança. Assim, a ausência de estímulo à independência dos filhos e a limitação da ação infantil, em seu ambiente, diminuem as possibilidades de enfrentamento de situações novas, as quais, ao longo do tempo, podem ser estressoras para as crianças. A restrição da autonomia dificultaria o acesso a reforçadores importantes para o aprendizado de novas habilidades essenciais para o desenvolvimento da criança. Bayer et al. (2006) afirmam ainda que, nesse contexto, as crianças aprendem a ter receio do ambiente e, assim, evitariam explorá-lo. Por outro lado, o controle comportamental está relacionado ao desenvolvimento social competente (Barber, 1996). Apesar dessas evidências, alguns achados parecem contrastantes. Os resultados do estudo longitudinal de Van der Bruggen et al. (2010), realizado quando as crianças tinham entre 3 e 5 anos, indicam apenas uma tendência positiva entre o controle psicológico materno e a ansiedade/depressão, assim como entre o envolvimento materno e a ansiedade/depressão ( $r=$ 0,$27 ; p<0,10 ; r=0,31 ; p=0,08$, respectivamente). McShane e Hastings (2009) não verificaram relações entre o suporte apropriado e os problemas internalizantes de pré-escolares. Por outro lado, no estudo de Feng, Shaw e Moilanen (2011), o controle psicológico materno, avaliado quando os filhos tinham entre 1 ano e meio e 2 anos, foi preditor de problemas internalizantes, avaliados entre os 6 e 7 anos de idade desses meninos.

Ainda assim, o uso predominante do controle psicológico pode ter um impacto importante quando se trata de interações com pré-escolares. Essa faixa etária, como afirmam Lamb, Hwang, Ketterlinus e Fracasso (1999, p. 416), é “marcada por mudanças desenvolvimentais dramáticas". Uma dessas é a crescente busca por autonomia, além da aquisição de novas habilidades físicas e cognitivas. É nesse período também que a criança começa a se relacionar com pares e seu ambiente extrapola os limites familiares, principalmente pela entrada na escola. Essas modificações exigem dos pais ou cuidadores novas formas de interação com os filhos, oferecendo segurança, mas permitindo a exploração do ambiente e o contato com novas experiências. A não sensibilidade a essas novas necessidades pode levar ao uso do controle psicológico e a prejuízos para o desenvolvimento social dos filhos tanto atual quanto futuro.

De forma geral, alguns estudos têm apontado as implicações negativas do controle psicológico para o desenvolvimento infantil, especialmente para os problemas internalizantes. Contudo, os achados ainda não são conclusivos, provavelmente devido ao número reduzido de estudos empíricos sobre os preditores dos problemas internalizantes (Bayer et al., 2010). Isso se agrava especialmente no contexto brasileiro, onde há uma grande lacuna na literatura a respeito dos problemas internalizantes e de seus principais preditores. Assim, o objetivo deste estudo foi verificar as relações entre o controle psicológico e o controle comportamental materno e os problemas internalizantes em crianças entre 3 e 5 anos. Foram examinadas também 
as relações entre características sociodemográficas das famílias e os problemas internalizantes das crianças. A hipótese principal foi de que os problemas internalizantes e suas síndromes estivessem positivamente correlacionados às duas dimensões do controle psicológico avaliadas (controle crítico e superproteção), assim como estariam negativamente correlacionados ao suporte apropriado, que é uma dimensão do controle comportamental.

\section{Método}

\section{Participantes}

Participaram do estudo 103 mães, com filhos de ambos os sexos (57,3\% de meninas e $54,7 \%$ de meninos), contatadas em sete escolas particulares de Salvador-BA, cujas mensalidades variaram entre $\mathrm{R} \$ 75,00$ e R \$220,00. A média de idade das crianças foi de 54,8 meses $(D P=9,2)$. As mães tinham idade média de 32,3 anos $(D P=6,8)$, estudaram, em média, 10,5 anos $(D P=2,8)$ e, das 103 participantes, $66 \%$ exerciam alguma atividade remunerada. Os pais das crianças tinham idade média de 35,8 anos $(D P=7,8)$ e aproximadamente 10 anos de escolaridade $(D P=2,6)$. Do total de mães, aproximadamente, $3 \%$ não souberam informar se o pai da criança exercia atividade remunerada e um dos pais já havia falecido. De acordo com $92,5 \%$ das mães, os

Tabela 1 Características Sociodemográficas das Participantes ( $N$ =103)

\begin{tabular}{lc}
\hline \multicolumn{1}{c}{ Característica } & $\begin{array}{c}\text { Média/Desvio } \\
\text { padrão/ Frequência }\end{array}$ \\
\hline Idade da mãe & $\mathrm{M}=32,3(\mathrm{DP}=6,8)$ \\
Escolaridade da mãe (anos) & $\mathrm{M}=10,54(\mathrm{DP}=2,8)$ \\
Mães que exercem atividade & $66 \%(68)$ \\
remunerada & $\mathrm{M}=35,8(\mathrm{DP}=7,8)$ \\
Idade do pai & $\mathrm{M}=10,1(\mathrm{DP}=2,6)$ \\
Escolaridade do pai (anos) & $92,2 \%(95)$ \\
Pais que exercem atividade & \\
remunerada & $65 \%(67)$ \\
Coabitação da mãe com pai da criança & $\mathrm{M}=3,9(\mathrm{DP}=1,3)$ \\
Número de moradores & \\
Renda familiar (em salários mínimos) & $31,1 \%(32)$ \\
Até 2 & $50,5 \%(52)$ \\
Acima de 2 até 4 & $7,8 \%(8)$ \\
Acima de 4 até 6 & $5,8 \%(6)$ \\
Acima de 8 até 10 & $1 \%(1)$ \\
$\quad$ Acima de 10 & \\
$\quad$ Sexo da criança & $57,3 \%(59)$ \\
Feminino & $42,7 \%(44)$ \\
\hline Idade da criança (em meses) & $\mathrm{M}=54,8(\mathrm{DP}=9,2)$ \\
\hline
\end{tabular}

pais da criança exerciam atividade remunerada, enquanto aproximadamente $4 \%$ dos pais estavam desempregados.

A residência dessas famílias tinha em média aproximadamente quatro moradores $(M=3,9 ; \mathrm{DP}=1,3)$, sendo que $65 \%$ dos pais moravam com a criança e a mãe da criança. Em relação à renda, 31,1\% das famílias recebiam até dois salários mínimos, $50,5 \%$ tinham renda entre dois e quatro salários mínimos e $13,6 \%$ tinham uma renda familiar mensal entre quatro e 10 salários mínimos. Apenas $1 \%$ das mães informou que a renda familiar era superior a 10 salários mínimos e, da mesma forma, apenas $1 \%$ não informou a renda familiar. A Tabela 1 apresenta os detalhes sobre as características sociodemográficas da amostra.

\section{Instrumentos}

Ficha de Dados Sociodemográficos: preenchida pelo pesquisador com as informações fornecidas pelas mães. Foram investigados dados sociodemográficos, como a idade, a escolaridade e a profissão dos pais, o estado civil materno, a renda familiar, entre outros.

Inventário de Comportamentos de Crianças entre 1 1/2 e 5 anos (Silvares, Rocha, \& Linhares, 2010): é a versão brasileira do Child Behavior Checklist for ages $11 / 2-5$ (CBCL - Achenbach \& Rescorla, 2000). O inventário avalia problemas de comportamento em crianças, entre 1 ano e meio e 5 anos, nos últimos dois meses de vida da criança, através do relato dos cuidadores. Neste estudo, os itens foram respondidos pelas mães. O inventário é composto por 100 itens que avaliam sete síndromes, sendo que quatro representam o agrupamento de problemas internalizantes (reatividade emocional, ansiedade/depressão, queixas somáticas e retraimento), duas representam o agrupamento de problemas externalizantes (problemas de atenção e comportamento agressivo), além da síndrome distúrbios do sono. Escores entre 60 e 63 e acima de 64, caracterizam uma amostra limítrofe e clínica na escala de problemas internalizantes, externalizantes e total de problemas emocionais/comportamentais. Escores abaixo de 60 são considerados não clínicos. Para as síndromes, escores até 64 são considerados não clínicos; entre 65 e 69 , limítrofe; e a partir de 70, clínicos. Os itens da versão original do CBCL apresentam alta consistência interna para todas as escalas, sendo 0,89 para a escala de problemas internalizantes, 0,96 para a escala de problemas externalizantes e 0,97 para a escala total de problemas. Contudo, ainda não há estudos de validação para a versão brasileira. No presente estudo, foi utilizada apenas a escala de problemas internalizantes e suas respectivas síndromes.

Entrevista Estruturada sobre Práticas Educativas Parentais e Socialização Infantil: é uma adaptação realizada por Lins, Oliveira, Palma e Alvarenga (2012) do The New Friends Vignettes (NFV -McShane \& Hastings, 2009). A entrevista busca verificar as reações dos pais frente a respostas tímidas dos filhos ao interagir com pares e é composta por duas situações hipotéticas, nas quais pais e filhos encontram outros pais e filhos. As situações são seguidas por afirmativas que descrevem possíveis ações dos pais, avaliando o uso do controle comportamental (suporte apropriado) e do 
controle psicológico (controle crítico e superproteção). Cada subescala (suporte apropriado, controle crítico e superproteção) é composta por 12 itens. O suporte apropriado é abordado por itens que refletem estratégias que facilitam a interação social dos filhos (ex. "Diga 'oi' para Juliana, filha"). O controle crítico é avaliado a partir de itens que abrangem a rejeição parental e o uso de críticas para ridicularizar ou insultar os filhos (ex. "Olha o que eu passo com essa menina."). Por fim, os itens de superproteção se referem às respostas parentais caracterizadas pelo envolvimento excessivo ou não necessário, pela restrição da autonomia e pela não estimulação de interações sociais (ex. "Pegaria ela/ele no colo e daria um abraço gostoso"). Para cada situação, as mães foram solicitadas a responder se adotariam as mesmas ações descritas nas afirmativas, caso passassem por situações semelhantes, de acordo com uma escala de três pontos: não, talvez e sim. Os escores das subescalas foram obtidos através do somatório das respostas, podendo variar de zero a 24. De acordo com o estudo de McShane e Hastings (2009), as subescalas que compõem o instrumento obtiveram bons índices de consistência interna, sendo 0,80 para o suporte apropriado, 0,75 para o controle crítico e 0,76 para a superproteção. Neste estudo, os coeficientes Alpha para o suporte apropriado, o controle crítico e a superproteção foram, respectivamente, 0,$72 ; 0,70$ e 0,69 .

\section{Procedimentos}

No presente estudo, foi utilizado um delineamento correlacional (Sampieri, Collado, \& Lucio, 2006) para a análise das relações entre o controle psicológico e comportamental materno e os escores de problemas internalizantes. Escolas particulares de Salvador foram contatadas e o projeto de pesquisa apresentado à direção da escola, sendo que sete escolas permitiram que o estudo fosse apresentado aos pais ou aos responsáveis dos alunos. $\mathrm{O}$ estudo foi divulgado através de convites enviados pela agenda escolar das crianças, de cartaz informativo afixado no mural das escolas, e através do contato direto dos pesquisadores com pais e responsáveis nos horários de chegada e saída das crianças. Posteriormente, as entrevistas foram agendadas com as mães que concordaram em participar, na data e no horário de sua preferência. Primeiramente, foi apresentado às mães o Termo de Consentimento Livre e Esclarecido, o qual foi assinado em duas vias. Em seguida, as mães responderam à Ficha de Dados Sociodemográficos, ao CBCL e à Entrevista Estruturada sobre Práticas Educativas Parentais e Socialização Infantil (McShane \& Hastings, 2009). Todos os instrumentos foram aplicados em formato de entrevista, ou seja, o pesquisador lia os itens para as participantes e estas, com auxílio de cartões de respostas, indicavam a alternativa escolhida. As entrevistas duraram aproximadamente 40 minutos cada. O presente estudo foi aprovado pelo Comitê de Ética em Pesquisa do Instituto de Saúde Coletiva da Universidade Federal da Bahia, em 28 de Junho de 2012 (Parecer no 027-12/CEP-ISC) e está de acordo com as normas de pesquisa envolvendo seres humanos.

\section{Resultados}

Através de análises descritivas, verificou-se que 28,2\% das crianças obtiveram classificação limítrofe ou clínica na escala de problemas internalizantes. Com o objetivo de examinar as relações entre os problemas internalizantes e as características sociodemográficas das participantes, foi utilizado o teste de correlação de Pearson. A escala de problemas internalizantes esteve negativamente correlacionada à idade materna $(r$ $=-0,26 ; p<0,01)$ e à idade paterna $(r=-0,27 ; p<0,01)$, assim como ao número de irmãos $(r=-0,22 ; p<0,05)$. Esses achados indicam que quanto mais jovem a mãe, quanto mais jovem o pai e quanto menor o número de irmãos, maiores os escores de problemas internalizantes. Da mesma forma, a síndrome reatividade emocional esteve negativamente correlacionada à idade materna $(r=-0,19 ; p<0,05)$, à idade paterna $(r=-0,18 ; p<0,05)$ e ao número de irmãos $(r=-0,16 ; p<0,05)$. Houve uma correlação negativa entre a síndrome ansiedade/depressão e a idade materna $(r=$ $0,24 ; p<0,01)$ e a idade paterna $(r=-0,24 ; p<0,01)$, e o número de irmãos $(r=-0,17 ; p<0,05)$. Foram verificadas também correlações negativas entre a síndrome ansiedade/ depressão e a idade materna $(r=-0,24 ; p<0,01)$ e a idade paterna $(r=-0,24 ; p<0,01)$, e o número de irmãos $(r=$ $0,17 ; p<0,05)$. Por fim, a síndrome queixas somáticas esteve negativamente correlacionada à idade materna $(r=-0,16 ; p<$ $0,05)$, enquanto a síndrome retraimento esteve negativamente

Tabela 2 Correlações (Pearson) entre os Problemas Internalizantes, as Respectivas Síndromes e as Características Sociodemográficas

\begin{tabular}{|c|c|c|c|c|c|}
\hline Características sociodemográficas & $\begin{array}{c}\text { Problemas } \\
\text { internalizantes }\end{array}$ & $\begin{array}{c}\text { Reatividade } \\
\text { emocional }\end{array}$ & $\begin{array}{c}\text { Ansiedade/ } \\
\text { Depressão }\end{array}$ & $\begin{array}{c}\text { Queixas } \\
\text { somáticas }\end{array}$ & Retraimento \\
\hline Idade materna & $-0,26^{* *}$ & $-0,19 *$ & $-0,24 * *$ & $-0,16^{*}$ & $-0,14$ \\
\hline Escolaridade materna & $-0,09$ & $-0,07$ & $-0,10$ & 0,12 & $-0,17 *$ \\
\hline Idade paterna & $-0,27^{* *}$ & $-0,18^{*}$ & $-0,24 * *$ & $-0,13$ & $-0,15$ \\
\hline Escolaridade paterna & 0,89 & $-0,06$ & 0,11 & 0,09 & 0,07 \\
\hline Idade da criança & 0,02 & 0,02 & 0,01 & 0,03 & $-0,01$ \\
\hline Número de irmãos & $-0,22^{*}$ & $-0,16^{*}$ & $-0,17^{*}$ & $-0,15$ & $-0,13$ \\
\hline Número de moradores & $-0,02$ & 0,08 & 0,05 & $-0,12$ & $-0,01$ \\
\hline
\end{tabular}

Nota: As variáveis idade paterna e escolaridade paterna possuem, respectivamente 99 e 96 casos válidos, visto que algumas mães não souberam informar tais dados a respeito dos pais das crianças e um dos pais já havia falecido.

$* p<0,05 ; * * p<0,01$ 
correlacionada à escolaridade materna $(r=-0,17 ; p<0,05)$. Os detalhes dessas análises são apresentados na Tabela 2.

Foram também conduzidas análises para avaliação de possíveis diferenças nos escores dos problemas internalizantes, considerando as variáveis sociodemográficas categóricas, como o sexo da criança, a ocupação da mãe e a coabitação parental. Para essas análises, foi utilizado o teste $\mathrm{t}$ de Student, contudo nenhuma diferença nos escores da escala de problemas internalizantes e das síndromes foi verificada, considerando essas variáveis. Apenas para a síndrome ansiedade/depressão, houve uma diferença entre as crianças cujos pais moravam e aquelas cujos pais não moravam com as mães das crianças e as crianças $[t(101)=$ - 2,43; $p=0,02]$. Os escores de ansiedade/depressão foram maiores para aquelas crianças cujos pais não moravam na mesma residência $(M=59,83 ; D P=7,6)$ em comparação aos escores das crianças cujos pais moravam na mesma residência $(M=56,36 ; D P=6,5)$.

A análise das correlações entre o controle materno e os problemas internalizantes foi realizada através do teste de correlação de Pearson. A Tabela 3 apresenta os coeficientes de correlação entre o escore total de problemas internalizantes, os escores das síndromes e os escores das dimensões do controle materno: suporte apropriado, controle crítico e superproteção. O controle crítico esteve positivamente correlacionado à ansiedade/depressão $(r=0,18 ; p<0,05)$ e ao retraimento $(r=0,20 ; p<0,05)$, entretanto, não foram encontradas correlações significativas entre as demais dimensões do controle materno e o total de problemas internalizantes, nem com as demais síndromes que compõem essa escala.

\section{Discussão}

O presente estudo teve como objetivo verificar as relações entre o controle materno (psicológico e comportamental) e os escores de problemas de comportamento internalizantes em pré-escolares. Os resultados obtidos não confirmaram as hipóteses do estudo, visto que a escala de problemas internalizantes não esteve correlacionada a nenhuma das dimensões do controle materno. Entretanto, a análise das síndromes que representam o agrupamento de problemas internalizantes mostrou que a ansiedade/depressão e o retraimento estiveram positivamente correlacionados ao controle crítico.

A correlação positiva significativa entre a ansiedade/ depressão e o controle crítico confirma alguns achados da literatura. Feng, Shaw e Silk (2008), por exemplo, afirmam que em relação à interação entre pais e filhos, o controle crítico tem sido relacionado de forma consistente ao desenvolvimento de ansiedade na criança. Em metanálises, realizadas por McLeod, Weisz, et al. (2007) e por McLeod, Wood, et al. (2007), a hostilidade foi uma das características parentais que se correlacionaram de forma positiva à depressão e à ansiedade infantil, sendo que, em comparação à ansiedade, a depressão foi explicada, em maior proporção, pela hostilidade parental. Além disso, nesses estudos, a ansiedade e a depressão estiveram negativamente correlacionadas ao suporte apropriado que se refere ao envolvimento adequado e ao incentivo à autonomia da criança.

Em relação à síndrome retraimento, os resultados confirmam os achados de Mills e Rubin (1998) e de Rubin, Burgess e Hastings (2002). Mills e Rubin, com o objetivo de verificar se o controle psicológico (ex. desvalorização da criança e não responsividade) e o controle comportamental (ex. monitoramento, uso de recompensas e comandos) estariam associados da mesma forma ou não à agressividade e ao retraimento social infantil, constataram que as mães de crianças que apresentavam retraimento social adotavam com mais frequência o controle psicológico e o comportamental, em comparação às mães de crianças agressivas. É importante destacar que o controle comportamental, nesse caso, era adotado de forma exagerada e inadequada. Rubin et al. avaliaram, através de observações da interação entre a mãe e a criança, em situações de brincadeira, com e sem a presença de pesquisadores, se a inibição comportamental, uma característica de temperamento, aos 2 anos seria um preditor de problemas internalizantes e retraimento aos 4 anos. Segundo os achados do estudo, a inibição entre pares verificada aos 2 anos de idade das crianças foi preditora de retraimento social aos 4 anos, quando as mães eram mais superprotetoras, faziam uso de insultos e ironias e zombavam dos filhos. Os autores concluíram que o controle crítico, além de limitar as oportunidades de crianças, que já apresentam vulnerabilidades para melhorar suas habilidades, afeta a confiança da criança em si, pois nesses casos a interação mãecriança é marcada por uma falta de confiança na capacidade dos filhos para desenvolver novas habilidades e para se comportar adequadamente. Assim, as crianças tenderiam a evitar interações com pares e com outras pessoas, isolandose ainda mais, o que ao longo do tempo pode estar também relacionado ao desenvolvimento de ansiedade e depressão, pelo acesso limitado a reforçadores sociais.

Da mesma forma que no presente estudo, McShane e Hastings (2009) não verificaram relações entre os problemas internalizantes e o suporte apropriado. Esses autores verificaram apenas que as professoras relataram menos problemas internalizantes em meninas, cujos pais, e não mães, utilizavam mais o suporte apropriado. Por sua vez,

Tabela 3 Correlações (Pearson) entre os Problemas Internalizantes, as Respectivas Síndromes e as Dimensões do Controle Materno

\begin{tabular}{lccccr}
\hline Dimensões do Controle Materno & $\begin{array}{c}\text { Problemas } \\
\text { internalizantes }\end{array}$ & $\begin{array}{c}\text { Reatividade } \\
\text { emocional }\end{array}$ & $\begin{array}{c}\text { Ansiedade/ } \\
\text { Depressão }\end{array}$ & $\begin{array}{c}\text { Queixas } \\
\text { somáticas }\end{array}$ & $\begin{array}{c}\text { Retraimento } \\
\text { Suporte apropriado }\end{array}$ \\
Controle Crítico & $-0,02$ & $-0,11$ & $-0,05$ & 0,02 & 0,06 \\
Superproteção & 0,12 & 0,09 & $0,18^{*}$ & 0,00 & $0,20^{*}$ \\
\hline
\end{tabular}

Nota: $* p<0,05$; 
os estudos de Bayer e colaboradores (Bayer et al., 2010, 2006, 2009), dos quais participaram predominantemente as mães, verificaram que o pouco envolvimento e o suporte parental foram preditores de problemas internalizantes, da mesma forma que a superproteção. Ainda assim, Mills et al. (2012) questionam se o suporte apropriado exerceria alguma influência sobre o desenvolvimento de problemas internalizantes, independentemente da influência de práticas negativas ou se esses dois tipos "seriam extremidades opostas de um mesmo contínuo” (p. 231). Nesse sentido, McShane e Hastings, apesar de afirmarem que o suporte apropriado seria um fator protetivo, principalmente quando as crianças apresentam vulnerabilidades para o desenvolvimento de problemas internalizantes, como algumas características de temperamento, sugerem que mais estudos devem ser desenvolvidos de forma que as condições nas quais o suporte apropriado contribui para um adequado desenvolvimento infantil sejam compreendidas.

No que diz respeito à influência de características sociodemográficas sobre os problemas internalizantes, algumas variáveis se destacaram. Os resultados indicam que quanto mais jovens as mães e os pais, maiores foram os escores na escala de problemas internalizantes, reatividade emocional e ansiedade/depressão, sendo que a idade materna ainda se correlacionou negativamente aos escores de queixas somáticas. Nesse sentido, Javo, Rønning, Heyerdahl e Rudmin (2004) constataram que quanto mais novas as mães participantes em seu estudo, maiores foram os escores de problemas internalizantes das crianças. É possível que pais mais jovens e com um número menor de filhos tenham mais dificuldades para interagir com os filhos, devido a um repertório comportamental mais limitado em comparação a pais mais velhos e com mais filhos. A experiência adquirida com outros filhos pode favorecer a aquisição de conhecimento a respeito do desenvolvimento infantil e o manejo, principalmente de comportamentos inadequados dos filhos. Além disso, é provável que filhos de pais mais jovens estejam expostos à influência de eventos estressores (ex. menor estabilidade financeira), que, por sua vez, afetariam os comportamentos parentais.

De acordo com os achados deste estudo, também não foram verificadas diferenças significativas nos escores de problemas internalizantes, considerando o sexo da criança. Esse achado corrobora os estudos de Bayer e colaboradores (Bayer et al., 2010, 2009), realizados com crianças em idade pré-escolar e escolar, assim como o estudo de Findlay, Coplan e Bowker (2009), que contou com a participação de 355 crianças com média de 10 anos e investigou as relações entre os relatos das crianças de timidez, problemas internalizantes e externalizantes e funcionamento social. Esses achados fortalecem a suposição de que as diferenças entre os sexos seriam mais evidentes durante a transição da infância para a adolescência, com as meninas apresentando maiores níveis médios e nítido aumento dos problemas internalizantes, ao longo dessa transição. Ao contrário, entre os meninos haveria maior estabilidade desses problemas de comportamento ao longo do desenvolvimento (Keiley et al., 2003; Leve, Kim, \& Pears, 2005).

A ausência de relações verificada entre o controle materno e a escala de problemas internalizantes aliada ao elevado número de crianças com classificação limítrofe ou clínica na escala de problemas internalizantes $(28,2 \%)$ produziram questionamentos a respeito da relevância do controle para a compreensão dos problemas internalizantes, assim como sobre as estratégias metodológicas utilizadas no presente estudo. Uma das hipóteses levantadas refere-se à possibilidade de as investigações sobre os problemas internalizantes terem herdado as concepções e os modelos dos estudos sobre os problemas externalizantes, mas que, no entanto, podem não ser apropriados ou suficientes para a compreensão dos problemas internalizantes e de suas síndromes. Granic e Lamey (2002), por exemplo, verificaram que mães de crianças que apresentavam comorbidade entre problemas internalizantes e externalizantes, além de adotarem práticas mais hostis, expressavam afeto negativo, através de postura e expressões faciais, assim como irritabilidade, enquanto para as mães de crianças que apresentavam apenas problemas externalizantes, o afeto durante a interação era neutro.

Nesse sentido, a forma como as mães ensinam os filhos a regular as suas emoções pode ser particularmente importante para os problemas internalizantes, visto que em comparação aos problemas externalizantes, os internalizantes envolvem predominantemente queixas de caráter emocional, como a ansiedade, a depressão e as preocupações excessivas. De acordo com Coyne e Thompson (2011), a presença de alguma psicopatologia, como, por exemplo, a depressão materna, pode ser particularmente importante para a compreensão das relações entre o afeto parental e o desenvolvimento de problemas internalizantes. Os autores verificaram que as mães em depressão estariam enfrentando dificuldades para lidar com suas emoções e poderiam ter mais dificuldades para atender às necessidades dos filhos. Como consequência, Coyne e Thompson sugerem que esses fatores prejudicariam o aprendizado infantil de como regular e lidar com as suas emoções, da mesma forma que poderiam prejudicar o estabelecimento de um apego seguro entre pais e filhos, fatores que contribuiriam para o desenvolvimento ou agravamento dos problemas internalizantes.

Contudo, deve-se atentar também para a diversidade de síndromes que compõem a escala de problemas internalizantes, o que pode dificultar a investigação dos principais preditores, visto que cada síndrome pode ter fatores etiológicos e trajetórias desenvolvimentais distintos. O controle crítico, por exemplo, esteve positivamente correlacionado à ansiedade/depressão e ao retraimento. Talvez essas duas síndromes sejam mais susceptíveis às influências dos comportamentos parentais característicos do controle crítico, como a hostilidade, a humilhação e as críticas excessivas. Assim, por exemplo, a restrição da autonomia da criança ou a punição das tentativas de exposição e de tomada de decisão, por parte da criança, contribuiriam para que a criança se tornasse retraída, como uma estratégia de esquiva de situações potencialmente aversivas causadas pelo controle crítico. Ao longo do desenvolvimento, esse tipo de controle poderia agravar sintomas de ansiedade/depressão, devido ao isolamento social e a outras consequências do retraimento, como o não desenvolvimento de habilidades necessárias para a interação com outras pessoas, que implicam na perda ou na falta de acesso a uma série de reforçadores. Por outro lado, as 
síndromes reatividade emocional e queixas somáticas podem estar ligadas a outras dimensões da interação mãe-criança.

Apesar desses achados, é importante destacar que o presente estudo apresentou algumas limitações. $\mathrm{O}$ controle materno foi acessado apenas a partir de como as mães imaginavam que reagiriam em situações semelhantes às apresentadas nas histórias. Talvez essa estratégia não seja uma forma sensível o suficiente para evocar as respostas das mães diante dos comportamentos dos filhos, principalmente para aquelas mães que nunca vivenciaram situações parecidas. É importante considerar que as situações apresentadas na entrevista ocorrem fora do contexto familiar, o que pode contribuir para que as mães, quando colocadas em situações como essas, não adotem o mesmo tipo de controle que adotariam no contexto familiar, de forma a evitar a avaliação negativa de outras pessoas. Ademais, as respostas obtidas podem ter sido influenciadas pela desejabilidade social, ou seja, as mães podem ter respondido a alguns itens do instrumento, considerando as respostas que são melhor avaliadas socialmente, evitando revelar a adoção de estratégias consideradas inadequadas, como, por exemplo, dizer que seu(a) filho(a) é bobo(a), na frente de outras pessoas. Outra limitação refere-se ao fato de o estudo contar apenas com as mães como informantes, tanto sobre o controle psicológico e comportamental, quanto sobre o comportamento infantil. A diversidade de informantes pode favorecer a coleta de informações mais precisas, por exemplo, no caso de mães depressivas, além de considerar as crianças em outros contextos e interações. Por fím, destaca-se o fato de que este estudo foi realizado com uma amostra de mães com características sociodemográficas semelhantes, todas residentes na cidade de Salvador. Isso limitaria a generalização dos achados deste estudo para outras populações, visto que os aspectos culturais têm implicações tanto para o controle materno quanto para as percepções das mães a respeito do comportamento de seus filhos (Darling \& Steinberg, 1993; Halgunseth, Ispa, \& Rudy, 2006). Dessa forma, futuras investigações devem ser realizadas, considerando essas limitações de forma que os achados deste estudo sejam ampliados.

\section{Considerações Finais}

O presente estudo examinou as relações entre o controle materno e os problemas internalizantes de préescolares, contudo, o impacto do controle psicológico não foi confirmado. É possível que outras variáveis, como as práticas de socialização emocional e a presença de alguma psicopatologia parental, sejam mais relevantes para a compreensão do surgimento e da manutenção dos problemas internalizantes.

De qualquer modo, o presente estudo aponta para a importância da investigação dos problemas internalizantes em crianças nos primeiros anos de vida, visto que os achados indicaram um elevado número de crianças com classificação limítrofe ou clínica nesta escala. Além disso, os resultados evidenciaram a relação entre o controle crítico, uma das dimensões do controle psicológico, que é caracterizado por hostilidade e humilhação, sobre certas síndromes que formam a escala de problemas internalizantes, e as relações entre essas síndromes e variáveis sociodemográficas, como a idade parental e o número de irmãos. A revisão da literatura realizada para este estudo indicou ainda uma quantidade muito limitada de instrumentos que avaliam o controle psicológico de pais de crianças no início da infância. Nesse sentido, o estudo oferece uma alternativa metodológica para a avaliação do controle parental, que foi adaptada com base em uma amostra baiana.

Por fim, considerando o número reduzido de estudos brasileiros sobre os problemas internalizantes e que esses problemas podem estar relacionados a importantes prejuízos no desenvolvimento infantil, salienta-se a necessidade de que novos estudos sejam elaborados, investigando, principalmente os seus preditores. Dessa forma, será possível o planejamento de estratégias de prevenção e intervenção precoces, que contribuam para a redução de problemas como a ansiedade e a depressão em outras fases da vida.

\section{Referências}

Achenbach, T. M. (1966). The classification of children's psychiatric symptoms: a factor-analytic study. Psychological Monographs: General and Applied, 80(7), 1-37. doi:10.1037/h0093906

Achenbach, T. M., \& Edelbrock, C. S. (1978). The classification of child psychopathology: a review and analysis of empirical efforts. Psychological Bulletin, 85(6), 1275-1301. doi:10.1037/0033-2909.85.6.1275

Achenbach, T. M., \& Rescorla, L. A. (2000). Manual for the ASEBA Preschool Forms \& Profiles. Burlington, VT: University of Vermont, Research Center for Children, Youth, \& Families.

Alvarenga, P. (2000). Práticas educativas maternas e problemas de comportamento na infância. (Unpublished master's thesis) Universidade Federal do Rio Grande do Sul, Porto Alegre.

Anselmi, L., Piccinini, C. A., Barros, F. C., \& Lopes, R. S. (2004). Psychosocial determinants of behaviour problems in Brazilian preschool children. Journal of Child Psychology and Psychiatry, 45(4), 779-788. doi:10.1111/j.1469-7610.2004.00271.x

Barber, B. K. (1996). Parental psychological control: Revisiting a neglected construct. Child Development, 67(6), 3296-3319. doi:10.1111/j.1467-8624.1996.tb01915.x

Bayer, J. K., Hastings, P. D., Sanson, A. V, Ukoumunne, O., \& Rubin, K. H. (2010). Predicting mid-childhood symptoms: a longitudinal community study. International Journal of Mental Health Promotion, 12(1), 16-28. doi:10.1080/14623 730.2010 .9721802

Bayer, J. K., Sanson, A. V, \& Hemphill, S. A. (2006). Parent influences on early childhood internalizing difficulties. Journal of Applied Developmental Psychology, 27(6), 542-559. doi:10.1016/j.appdev.2006.08.002

Bayer, J. K., Sanson, A. V, \& Hemphill, S. A. (2009). Early childhood aetiology of internalising difficulties: a longitudinal community study. International Journal of Mental Health Promotion, 11(1), 22-32. doi:10.1080/14623730.2009.9721777 
Caron, A., Weiss, B., Harris, V., \& Catron, T. (2006). Parenting behavior dimensions and child psychopathology: specificity, task dependency, and interactive relations. Journal of Clinical Child and Adolescent Psychology, 35(1), 34-45. doi:10.1207/ s15374424jccp3501_4

Cova, F., Maganto, C., \& Melipillán, R. (2005). Género, adversidad familiar y síntomas emocionales en preadolescentes. Psykhe (Santiago), 14(1), 227-232. doi:10.4067/S071822282005000100017

Coyne, L. W., \& Thompson, A. D. (2011). Maternal depression, locus of control, and emotion regulatory strategy as predictors of preschoolers' internalizing problems. Journal of Child and Family Studies, 20(6), 873-883. doi:10.1007/s10826-0119455-2

Crawford, T. N., Cohen, P., Midlarsky, E., \& Brook, J. S. (2001). Internalizing symptoms in adolescents: gender differences in vulnerability to parental distress and discord. Journal of Research on Adolescence, 11(1), 95-118. doi:10.1111/15327795.00005

Darling, N., \& Steinberg, L. (1993). Parenting style as context: an integrative model. Psychological Bulletin, 113(3), 487-496. doi:10.1037/0033-2909.113.3.487

Feng, X., Shaw, D., \& Moilanen, K. L. (2011). Parental negative control moderates the shyness-emotion regulation pathway to school-age internalizing symptoms. Journal of Abnormal Child Psychology, 39(3), 425-436. doi:10.1007/s10802-010-9469-z

Feng, X., Shaw, D., \& Silk, J. (2008). Developmental trajectories of anxiety symptoms among boys across early and middle childhood. Journal of Abnormal Psychology, 117(1), 32-47. doi:10.1037/0021-843X.117.1.32

Findlay, L. C., Coplan, R. J., \& Bowker, A. B. (2009). Keeping it all inside: shyness, internalizing coping strategies and socio-emotional adjustment in middle childhood. International Journal of Behavioral Development, 33(1), 4754. doi:10.1177/0165025408098017

Granic, I., \& Lamey, A. V. (2002). Combining dynamic systems and multivariate analyses to compare the mother-child interactions of externalizing subtypes. Journal of Abnormal Child Psychology, 30(3), 265-283. doi:0091-0627/02/0600-0265/0

Halgunseth, L. C., Ispa, J. M., \& Rudy, D. (2006). Parental control in Latino families: an integrated review of the literature. Child Development, 77(5), 1282-1297. doi:10.1111/j.14678624.2006.00934.X

Javo, C., Rønning, J. A., Heyerdahl, S., \& Rudmin, F. W. (2004). Parenting correlates of child behavior problems in a multiethnic community sample of preschool children in northern Norway. European Child \& Adolescent Psychiatry, 13(1), 8-18. doi:10.1007/s00787-004-0349-3

Keiley, M. K., Bates, J. E., Dodge, K. A., \& Pettit, G. S. (2000). A cross-domain growth analysis: externalizing and internalizing behaviors during 8 years of childhood. Journal of Abnormal Child Psychology, 28(2), 161-179. doi:10.1023/A:1005122814723

Keiley, M. K., Lofthouse, N., Bates, J. E., Dodge, K. A., \& Pettit, G. S. (2003). Differential risks of covarying and pure components in mother and teacher reports of externalizing and internalizing behavior across ages 5 to 14. Journal of Abnormal Child Psychology, 31(3), 267-283. doi:10.1023/A:1023277413027
Kovacs, M., \& Devlin, B. (1998). Internalizing disorders in childhood. Journal of Child Psychology and Psychiatry, 39(1), 47-63. doi:10.1111/1469-7610.00303

Lamb, M., Hwang, C. P., Ketterlinus, R. D., \& Fracasso, M. P. (1999). Parent-child relationships: development in context of the family. In M. H. Bornstein \& M. Lamb (Eds.), Developmental Psychology: an advanced text book (pp. 411450). Mahwah/London: LEA.

Leve, L. D., Kim, H. K., \& Pears, K. C. (2005). Childhood temperament and family environment as predictors of internalizing and externalizing trajectories from ages 5 to 17. Journal of Abnormal Child Psychology, 33(5), 505-520. doi:10.1007/s10802-005-6734-7

Lins, T., Oliveira, J. M., Palma, E., \& Alvarenga, P. (2012). Entrevista Estruturada sobre Práticas Educativas Parentais e Socialização Infantil. Manuscrito não publicado.

Marturano, E. M., Toller, G. P., \& Elias, L. C. S. (2005). Gênero, adversidade e problemas socioemocionais associados à queixa escolar. Estudos de Psicologia (Campinas), 22(4), 371-380. doi:10.1590/S0103-166X2005000400005

Mathiesen, K. S., Sanson, A. V, Stoolmiller, M., \& Karevold, E. (2009). The nature and predictors of undercontrolled and internalizing problem trajectories across early childhood. Journal of Abnormal Child Psychology, 37(2), 209-222. doi:10.1007/s10802-008-9268-y

McLeod, B. D., Weisz, J. R., \& Wood, J. J. (2007). Examining the association between parenting and childhood depression: a meta-analysis. Clinical Psychology Review, 27(8), 986-1003. doi:10.1016/j.cpr.2007.03.001

McLeod, B. D., Wood, J. J., \& Weisz, J. R. (2007). Examining the association between parenting and childhood anxiety: a meta-analysis. Clinical Psychology Review, 27(2), 155-172. doi:10.1016/j.cpr.2006.09.002

McShane, K. E., \& Hastings, P. D. (2009). The New Friends Vignettes: measuring parental psychological control that confers risk for anxious adjustment in preschoolers. International Journal of Behavioral Development, 33(6), 481495. doi:10.1177/0165025409103874

Mills, R. S. L., Hastings, P. D., Helm, J., Serbin, L. A., Etezadi, J., Stack, D. M., ... Li, H. H. (2012). Temperamental, parental, and contextual contributors to early-emerging internalizing problems: a new integrative analysis approach. Social Development, 21(2), 229-253. doi:10.1111/j.14679507.2011.00629.x

Mills, R. S. L., \& Rubin, K. H. (1998). Are behavioural and psychological control both differentially associated with childhood aggression and social withdrawal? Canadian Journal of Behavioural Science, 30(2), 132-136. doi:10.1037/ h0085803

Oland, A. A., \& Shaw, D. (2005). Pure versus co-occurring externalizing and internalizing symptoms in children: the potential role of socio-developmental milestones. Clinical Child and Family Psychology Review, 8(4), 247-270. doi:10.1007/s10567-005-8808-Z

Patterson, G., Reid, J., \& Dishion, T. (1992). Positive parenting and children's prossocial skills. In G. Patterson, J. Reid, \& T. Dishion (Eds.), Antisocial boys: a Social Interaction Approach (Vol. 4). Eugene, OR: Castalia Publishing Co. 
Peterson, D. R. (1961). Behavior problems of middle childhood. Journal of Consulting Psychology, 25(3), 205-209. doi:10.1037/ h0038994

Rubin, K. H., Burgess, K. B., \& Hastings, P. D. (2002). Stability and social-behavioral consequences of toddlers' inhibited temperament and parenting behaviors. Child Development, 73(2), 483-495. doi:10.1111/1467-8624.00419

Sampieri, R. H., Collado, C. F., \& Lucio, P. B. (2006). Metodologia de pesquisa. São Paulo, SP: McGraw-Hill.

Sawyer, M. G., Arney, F. M., Baghurst, P. A., Clark, J. J., Graetz, B. W., Kosky, R. J., ... Zubrick, S. R. (2001). The mental health of young people in Australia: key findings from the child and adolescent component of the national survey of mental health and well-being. The Australian and New Zealand Journal of Psychiatry, 35(6), 806-814. doi:10.1046/j.14401614.2001.00964.x
Silk, J., Shaw, D., Prout, J., O’Rourke, F., Lane, T., \& Kovacs, M. (2011). Socialization of emotion and offspring internalizing symptoms in mothers with childhood-onset depression. Journal of Applied Developmental Psychology, 32(3), 127136. doi:10.1016/j.appdev.2011.02.001

Silvares, E. F. M., Rocha, M. M., \& Linhares, M. B. M. (2010). Inventário de Comportamentos de Crianças entre 1 1/2 - 5 anos (CBCL/11/2-5). Versão brasileira do "Child Behavior Checklist for ages 1 1/2 -5." Manuscrito não publicado.

Van der Bruggen, C., Stams, G. J. J. M., Bogels, S. M., \& Paulussen-Hoogeboom, M. C. (2010). Parenting behaviour as a mediator between young children's negative emotionality and their anxiety/depression. Infant and Child Development, 19(4), 354-365. doi:10.1002/icd.665

Recebido em 23.08.2013 Primeira decisão editorial em 04.06.2014 Versão final em 02.07.2014 Aceito em 23.07.2014 